\title{
MECYSMAUCHENIUS SEGMENTATUS (ARANEAE: MECYSMAUCHENIIDAE) EN ISLA RIESCO: AMPLIACIÓN DE SU DISTRIBUCIÓN CONOCIDA EN MAGALLANES, CHILE
}

\author{
MECYSMAUCHENIUS SEGMENTATUS (ARANEAE: MECYSMAUCHENIIDAE) IN RIESCO \\ ISLAND: EXTENSION OF ITS KNOWN DISTRIBUTION IN MAGALLANES, CHILE
}

Andrés Taucare-Ríos ${ }^{1,2}$

La familia Mecysmaucheniidae Simon, 1895 comprende un grupo de arañas criptozoicas caracterizadas esencialmente por poseer sólo dos hileras y quelíceros enormemente alargados que surgen de una especie de foramen formado por los márgenes laterales del cefalotórax al unirse ventralmente (Forster \& Platnick, 1984). Los mecismauquénidos son arañas de distribución gondwánica, de bosques de Nueva Zelanda, centro y sur de Chile, y sur de la Argentina, incluyendo archipiélagos cercanos, como Juan Fernández y Malvinas (Grismado \& Ramírez, 2008). Usualmente se las ubica dentro de la superfamilia Palpimanoidea, grupo definido por sus características especiales en la morfología de los quelíceros: presencia de setas romas ("peg teeth") y de la glándula queliceral que se abre en un elevado tubérculo (Forster \& Platnick, 1984). En Sudamérica se conocen cinco géneros de estas arañas; Mecysmauchenius Simon, 1884 con diecisiete especies descritas, y los restantes cuatro, descritos por Forster y Platnick (1984) son: Mecysmauchenioides con dos especies,
Semysmauchenius, Mesarchaea y Chilarchaea con una especie cada uno (Grismado \& Ramírez, 2008; Platnick, 2013).

En Chile esta familia se encuentra actualmente representada por cinco géneros y 20 especies (Platnick, 2013). La mayor parte se encuentran en Tierra del Fuego, extremo sur de Chile (Grismado \& Ramírez, 2008). Una de las con distribución más amplia es Mecysmauchenius segmentatus Simon, 1884, siendo típica de los Bosques Magallánicos de Chile y Argentina (Grismado \& Ramírez, 2008). Previamente esta especie ha sido registrada en Chile para las localidades de Osorno, Malleco $y$ en distintas localidades del sector oriental de Magallanes : Rio Blanco (46 $\left.{ }^{\circ} 15^{\prime} \mathrm{S} ; 72^{\circ} 55^{\prime} \mathrm{W}\right)$, Isla Hoste $\left(55^{\circ} 15^{\prime} \mathrm{S} ; 69^{\circ} 0^{\prime} \mathrm{W}\right)$, Vicuña, Isla Grande (53 $56^{\prime} \mathrm{S}$; $\left.68^{\circ} 32^{\prime} \mathrm{W}\right)$, Oeste de Laguna Amarga $\left(50^{\circ} 45^{\prime} \mathrm{S} ; 7^{\circ} 22^{\prime} \mathrm{W}\right)$, Puerto Williams, Isla Navarino $\left(55^{\circ} 4^{\prime} \mathrm{S}\right.$; $67^{\circ} 40^{\prime} \mathrm{W}$ ) (Forster \& Platnick, 1984; Grismado \& Lopardo, 2003), sin embargo nunca antes ha sido reportada para Isla Riesco. La presente nota tiene como finalidad

\footnotetext{
${ }^{1}$ Programa de Doctorado en Ecología y Biología Evolutiva, Facultad de Ciencias, Universidad de Chile, and.taucare26@gmail.com ${ }^{2}$ Laboratorio de Conservación Biológica, Facultad de Ciencias, Las Palmeras 3425, Santiago, Universidad de Chile.
} 
entregar nuevos datos acerca de la distribución de esta especie en Chile y específicamente en la Región de Magallanes a partir del hallazgo de un ejemplar en isla Riesco, contribuyendo de esta manera al conocimiento de la biodiversidad de la región y del país .

Materialexaminado: $\mathrm{CHILE}$, IslaRiesco $\left(53^{\circ} 0^{\prime} \mathrm{S}\right.$; $\left.72^{\circ} 30^{\prime} \mathrm{W}\right), 800$ msnm, Región de Magallanes, Fecha: 12/05/13, 1 ô colectado manualmente. G.Stipicic y G. Simonetti leg. El ejemplar fue colectado en bosque de lenga (Nothofagus pumilio) sometido a pastoreo ovino. Para la identificación se siguió la descripción de Forster y Platnick (1984). Las fotografías fueron tomadas con una cámara digital Sony SteadyShot de 14 megapixeles en una lupa estereoscópica Leica S6E. El material colectado se encuentra depositado en el Museo Nacional de Historia Nacional (MNHN) en Santiago de Chile.

Descripción: Cefalotórax de color marrónanaranjado con área cefálica elevada, no presentando ninguna constricción entre las áreas cefálica y torácica. Abdomen dorsalmente con una banda púrpura transversal a través de la superficie anterodorsal, conectado medialmente a una serie de tres bandas púrpuras (Fig.1) que se extienden posteriormente hasta cerca de las hileras. Palpo (Fig. 2A-C) con un solo dentículo en el ápice de la protuberancia retrolateral de la tibia, este dentículo se ubica más proximalmente
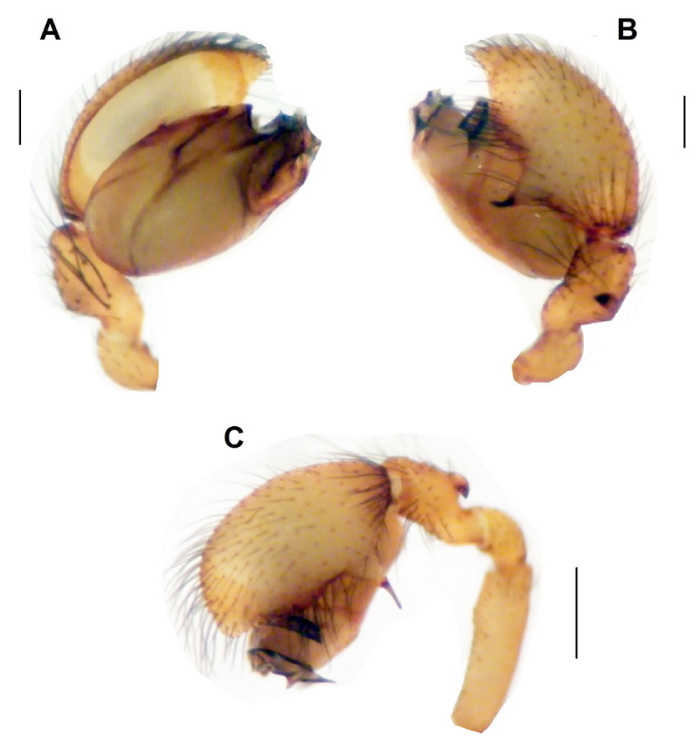

Fig. 2A-C. Palpo izquierdo del macho de Mecysmauchenius segmentatus A. Vista prolateral ; B and C. Vista retrolateral. Escala: $0,25 \mathrm{~mm}$ para A y B; $0,5 \mathrm{~mm}$ para $\mathrm{C}$.

en relación con las demás especies del género.

Comentarios: Los mecismauquénidos tienen hábitos criptozoicos, encontrándoselos generalmente en la hojarasca o bajo piedras en los bosques húmedos australes. El presente hallazgo corresponde el primer registro de esta especie para Isla Riesco, ampliándose su distribución dentro de la Region de Magallanes,

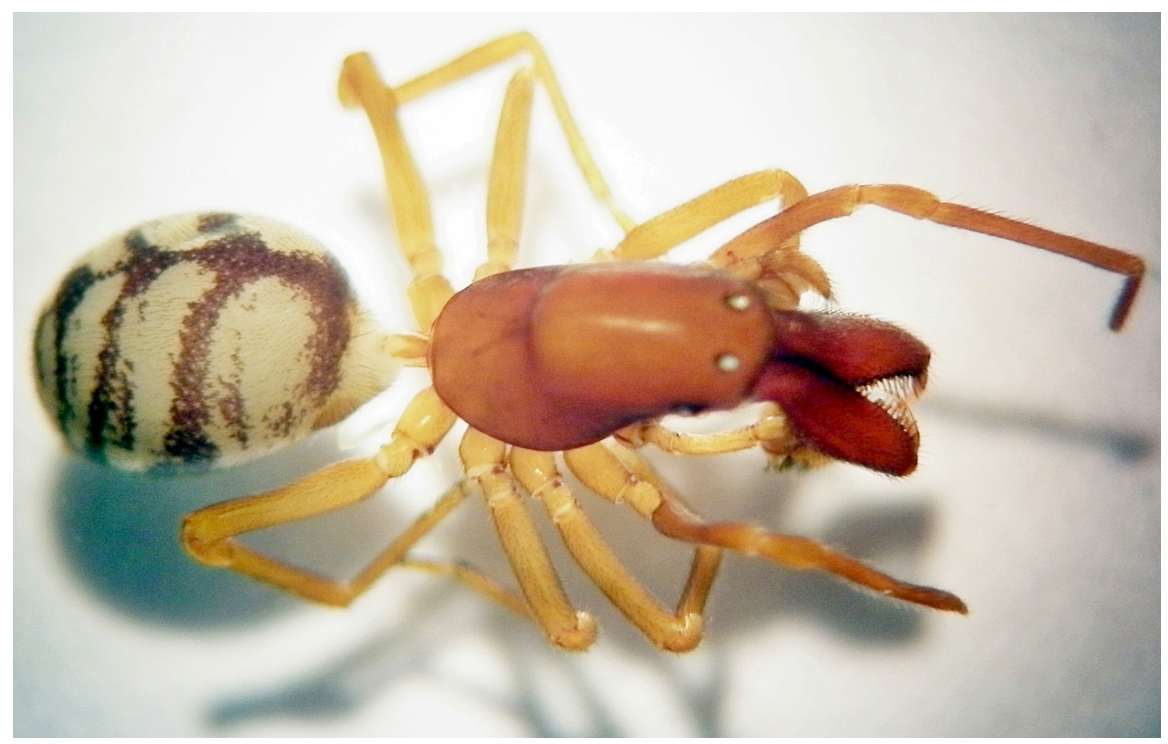

Fig. 1. Vista dorsal del macho de M. segmentatus, Isla Riesco, Región de Magallanes. 
por lo que de esta manera puede considerarse como un arácnido bastante frecuente del bosque subpolar magallánico, incluyendo los bosques sometidos a pastoreo.

\section{AGRADECIMIENTOS}

Quisiera agradecer profundamente el aporte realizado por el Dr. Norman Platnick en el presente manuscrito, facilitando parte de la biografía utilizada y atendiendo gentilmente a nuestras dudas e inquietudes respecto a esta interesante especie. Agradezco a G. Stipicic y G. Simonetti (Estancia Anita Beatriz, Isla Riesco) por proveernos las muestras de arácnidos de Isla Riesco. Finalmente agradezco de manera muy especial al Dr. Javier Simometti por sus valiosos comentarios y sugerencias en este trabajo.

\section{LITERATURA CITADA}

Forster, R. R. \& Platnick, N. I. (1984). A review of the archaeid spiders and their relatives, with notes on the limits of the superfamily Palpimanoidea (Arachnida: Araneae). Bulletin American Museum Natural History, 178(1), 1-106.
Grismado, C. J. \& L. Lopardo (2003). Nuevos datos sobre la distribución geográfica de las familias australes de arañas Malkaridae y Mecysmaucheniidae (Arachnida: Araneae), con la descripción de la hembra de Mecysmauchenius thayerae Forster \& Platnick. Revista Ibérica de Aracnología 8, 37-43.

Grismado, C. J. \& M. J. Ramírez (2008). Mecysmaucheniidae. En: Claps, L. E.; G. Debandi \& S. Roig-Juñent, (Dirs.). Biodiversidad de Artrópodos Argentinos, Vol. 2, 91-95 págs. Editorial Sociedad Entomológica Argentina, Mendoza.

Grismado, C. J. \& L. Lopardo (2003). Nuevos datos sobre la distribución geográfica de las familias australes de arañas Malkaridae y Mecysmaucheniidae (Arachnida: Araneae), con la descripción de la hembra de Mecysmauchenius thayerae Forster \& Platnick. Revista Ibérica de Aracnología 8, 37-43.

Platnick, N.I. (2013). The World Spider Catalog. Version 13.5. American Museum of Natural History. http://research.amnh. org/entomology/spiders/catalog/ (assessed 10.IX.2012). 
